\title{
Line Profile microvariability and wind structure for OB stars
}

\author{
Alexander F. Kholtygin ${ }^{1}$, Sergei N. Fabrika ${ }^{2}$, and Natalia P. Sudnik ${ }^{1}$ \\ ${ }^{1}$ Astronomical Institute, Saint-Petersburg University, 198504, Petrodvoretz, Russia \\ email: afkholtygin@gmail.com \\ ${ }^{2}$ Special Astrophysical Observatory, Nizhnii Arxyz, Russia
}

\begin{abstract}
We report the results of a search for line profile variability (LPV) in the spectra of OB stars. The wavelets were used for looking for the irregular LPV in spectra of program stars. We connect the appearance of irregular details in the LPV with the formation and dissipation of the small-scale substructures (clumps) in the wind.
\end{abstract}

Keywords. stars: early-type, stars: variables: other, line: profiles, stars: winds, outflows

\section{Line profile variability}

Our program of searching for small amplitude microvariations of the line profiles (microLPV) in spectra of OB stars had started in 2001 (Kholtygin et al. 2003). The observations were made with the $1.8 \mathrm{~m}$ telescope of the Korean Bohyunsan Optical Astronomical Observatory and 1-m and 6-m telescopes of the Special Astrophysical Observatory, Russia. More than 1000 spectra of 12 OB stars were obtained. Both regular and stochastic microLPV in spectra of all program stars are found.

For the detection of microLPV of various nature we use the smooth Time Variation Spectra (smTVS, see Kholtygin et al. 2006 for details):

$$
\operatorname{smTVS}(\lambda, S)=\left[\frac{1}{N-1}\left(\sum_{i=1}^{n}\left[F_{i}(\lambda, S)-\overline{F(\lambda, S)}\right]^{2}\right)\right]^{1 / 2},
$$

where $F_{i}(\lambda, S)$ is the flux in a line at wavelength $\lambda$, smoothed with a Gaussian filter with width $S, i$ the number of the spectrum, $N$ the total number of spectra, and $\overline{F(\lambda, S)}$ the mean of all smoothed line fluxes. For $S=0$ the $\operatorname{sm} T V S(\lambda, 0)$ spectra corresponds to the Time Variation Spectra introduced by Fullerton et al. (1996). The smTVS spectrum for the HeII 4200 line in a spectrum of $\lambda$ Cep is plotted in Fig. 1 (left panel). The microLPV for these lines is seen at all widths. For values of $S>1 \AA$ we can see a very weak LPV of the line CIII 4187, which cannot be detected by other methods. In the Fourier spectrum of LPV in spectra of $\lambda$ Cep there is a wide peak at $\nu=3.46 \pm 0.3 d^{-1}$. This can be explained by non-radial photospheric pulsations with a period $P=6.9 \pm 0.6 h$.

\section{Wind structure}

As a tool to detect the microdetails in a line profile connected with small clumps in the wind we use the dynamical wavelet spectra of residuals of the line profile (see Kholtygin et al. 2006 for details):

$$
\mathcal{W}_{t}(s, V, t)=\frac{1}{\sqrt{S}} \int_{-\infty}^{\infty} f(V, t) \psi\left(\frac{x-V}{S}\right) d x
$$



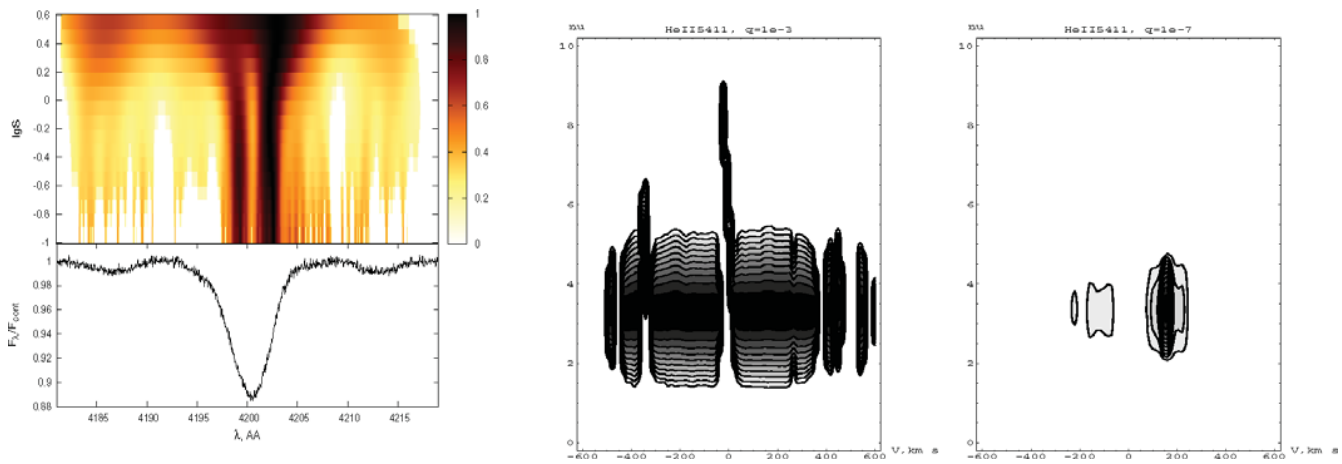

Figure 1. Left Panel: smTVS spectra for HeII $\lambda 4200$ line in spectra of $\lambda$ Cep (top) and mean line profile (bottom). Right Panel: Fourier-spectra of the line HeII $\lambda 5411$ LPV in spectra of $\lambda$ Cep for FAP level $q=10^{-3}$ (left) and $q=10^{-7}$ (right).
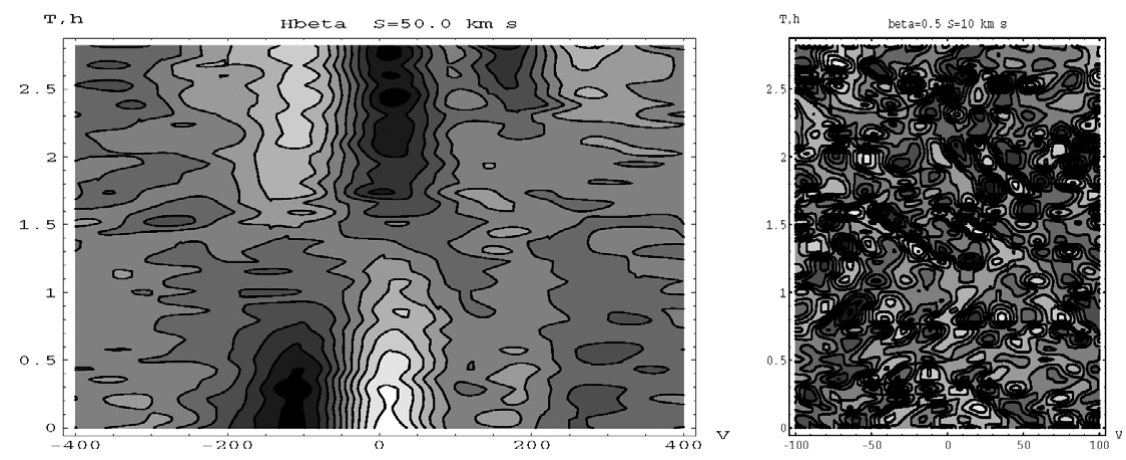

Figure 2. Left Panel: Dynamical wavelet spectra for line $\mathrm{H}_{\beta}$ in spectra of $\delta$ Ori A for the velocity interval $-400 \mathrm{~km} / \mathrm{s} \leqslant V \leqslant 400 \mathrm{~km} / \mathrm{s}$ and a scale $S=50 \mathrm{~km} / \mathrm{s}$. The time is counted in hours from beginning of observations. Right Panel: The same as in the Left Panel, but for $S=10 \mathrm{~km} / \mathrm{s}$ and $-100 \mathrm{~km} / \mathrm{s} \leqslant V \leqslant 100 \mathrm{~km} / \mathrm{s}$.

where $f(V, t)$ is an analysed function. In our case $f(V, t)=F(V, t)-\overline{F(V, t)}$. Here $F(V, t)$ is an individual line profile measured at the moment $t, \overline{F(V, t)}$ is the mean profile averaged over all observations. For the sake of convenience we use the Doppler shift $V$ from the line centre instead of wavelength $\lambda$. In this case the scale $S$ of the wavelet transform is expressed in $\mathrm{km} / \mathrm{s}$. As a mother wavelet we exploit the MHAT-wavelet $\psi(x)=(1-$ $\left.x^{2}\right) \exp \left(-x^{2} / 2\right)$.

In Fig. 2 we present the dynamical wavelet spectra of LPV in the line $\mathrm{H}_{\beta}$ in spectra of $\delta$ Ori A for scales $S=50 \mathrm{~km} / \mathrm{s}$ (left panel) and $S=10 \mathrm{~km} / \mathrm{s}$ (right panel). For the larger scale $S=50 \mathrm{~km} / \mathrm{s}$ we see in the dynamical wavelet spectrum mainly regular LPV, connected with non-radial pulsations with a period $P \approx 4^{\mathrm{h}}$ (see Kholtygin et al. 2006). At the same time, the numerous irregular details in the dynamical wavelet spectra for $S=10 \mathrm{~km} / \mathrm{s}$ can be connected with small clumps in the wind of the main component $\mathrm{Aa}^{1}$ of the triple system $\delta$ Ori A, having the velocity dispersion $\sigma_{V}=10-20 \mathrm{~km} / \mathrm{s}$.

\section{References}

Kholtygin, A. F., Monin, D. N., Surkov, A. E., \& Fabrika, S. N. 2003, Astron. Lett., 29, 175

Kholtygin, A. F., Burlakova, T. E., Fabrika, S. N., Valyavin, G. G. et al. 2006, Astron. Rep., 50, 887

Fullerton, A. W., Gies, D. R., \& Bolton, C. T. 1996, ApJS, 103, 475 